\section{O Uso da Ultra-Sonografia na Avaliação da Distribuição de Gordura Abdominal}

\begin{abstract}
RESUMO
A quantificação da adiposidade visceral é de suma importância, pois a gordura visceral é a grande responsável pelas complicações metabólicas da população obesa. O método de escolha para tal quantificação é a Tomografia Computadorizada. No entanto, este exame tem alto custo, é pouco prático e submete os individuos aos riscos da irradiação. A medida de cintura, a relação cintura-quadril e o diâmetro sagital são métodos que determinam indiretamente a gordura visceral. A ultra-sonografia tem sido proposta como uma técnica não invasiva para a avaliação de gordura intra-abdominal. No presente estudo foram determinadas, através da ultra-sonografia. as espessuras subcutâneas e intra-abdominais em 29 mulheres obesas em pré-menopausa. Estes valores foram comparados com os parâmetros antropométricos e com as áreas subcutâneas e viscerais medidas pela tomografia computadorizada. A espessura intraabdominal foi a variável que obteve maior coeficiente de correlação com as áreas adiposas viscerais. Para a equação preditiva de área visceral, além da espessura intra-abdominal, foram incluídas as variáveis espessura subcutânea e medida de cintura. A espessura intra-abdominal mostrou correlação significativa com os niveis tensionais e com os valores de triglicerídeos. A correlação entre a ultrasonografia e a tomografia computadorizada foi maior no grupo onde as áreas viscerais eram maiores. A ultra-sonografia é um método útil para a determinação do tecido adiposo visceral. (Arq Bras Endocrinol Metab 2000; 44/1: 5-12)
\end{abstract}

Unitermos: Obesidade visceral; Diagnóstico; Ultra-sonografia artigo original

\author{
Rosana B. Radominski \\ Denise P. Vezozzo \\ Giovanni G. Cerri \\ Alfredo Halpern
}

\begin{abstract}
The measurement of visceral adipose tissue is very important as the visceral fat plays a major role in the metabolic disorders of the obese people. Computed tomography is the reference method for intraabdominal fat evaluation, but it is expensive, with fairly limited availability and employs ionizing radiations. Waist circumference, waist/hip ratio and sagittal diameter are considered representative measurements of visceral fat. However they remain indirect means for calculating intra-abdominal adipose deposits. Sonography has been proposed as a noninvasive technique for measuring visceral fat. In this study subcutaneous and intra-abdominal thickness were measured by sonography on 29 premenopausal obese females. The results were compared with the anthropometric methods and with the subcutaneous and visceral adipose tissue areas measured by computed tomography. Uitrasound intra-abdominal thickness was the variable with highest correlation coefficient with visceral fat areas. In the predictive equations for visceral adipose tissue area besides intra-abdominal thickness, subcutaneous fat thickness and waist circumference was included. The intra-abdominal thickness
\end{abstract}

\author{
Grupo de Obesidade - \\ Disciplina de Endocrinologia da \\ Universidade de São Paulo, SP.
}


showed significant correlations with the blood pressure level and triglyceride level. The correlation between sonography and computed tomography was highest as visceral fat area increased. Sonography is a useful method for measuring visceral adipose tissue. (Arq Bras Endocrinol Metab 2000; 44/1: 5-12)

Keywords: Visceral obesity; Diagnosis; Sonography

A OBESIDADE É UMA DOENÇA CRÔNICA que está associada a uma série de patologias como dislipidemias, doenças cardiovasculares, hipertensão arterial, diabetes melito, colecistopatia, alguns tipos de, e com efeitos diretos e indiretos sobre, a morbidade e mortalidade nas mais diversas populações (1-7).

Ao longo dos anos, pesquisas vêm demonstrando que o aumento do peso por si só é menos relevante do que a distribuição do tecido adiposo corporal na determinação das alteraçóes metabólicas. Em 1956, o Professor Jean Vague (8), da Universidade de Marselha, associou a obesidade do tipo andróide (deposição de tecido adiposo em região de tronco) à maior incidência de diabetes melito, gota e aterosclerose. Mais de 30 anos após, estudos clínicos e epidemiológicos, utilizando medidas antropométricas como a Relação Cintura-Quadril (RCQ), vieram a confirmar os achados iniciais de Vague (9-11).

Recentemente, o desenvolvimento de técnicas mais sofisticadas para a avaliação de gordura corporal tornou evidente que a morbidade, a mortalidade, bem como as alterações metabólicas nos obesos, estão mais relacionadas com a quantidade de tecido adiposo visceral do que com a gordura corporal total (12-18). Atualmente, a tomografia computadorizada é considerada o método de referência para a avaliação de gordura intra-abdominal (19). Entretanto é um método dispendioso, que submete os pacientes a radiação ionizante c pouco disponível.

Considerando a importância da gordura intraabdominal como fator determinante de alterações metabólicas associadas à obesidade, a substituição da TC por um método mais simples, de baixo custo $\mathrm{e}$ livre de irradiação, significa um avanço no diagnóstico da obesidade visceral. Amparado nestas convicçōes, o presente trabalho objetivou principalmente avaliar a eficácia da US na determinação de gordura intraabdominal em mulheres obesas brasileiras que não atingiram a menopausa.

\section{CASUÍSTICA E MÉTODOS}

Para a execução deste trabalho, foram estudadas 29 mulheres na pré-menopausa, com idade entre 16 e $\mathbf{5 0}$ anos e IMC entre 24,07 e $37,45 \mathrm{~kg} / \mathrm{m}^{2}$, recrutadas entre as que procuraram o Ambulatório de Obesidade do serviço de Endocrinologia do Hospital de Clínicas da Faculdade de Medicina da Universidade de São Paulo, no período entre março de 1994 e dezembro de 1995.

Após assinarem o consentimento informado, todas as pacientes foram submetidas à anamnese e ao exame clínico. Nenhuma delas apresentava diabetes melito ou dislipidemia diagnosticados ou hipertensão arterial grave.

As medidas de pressão arterial sistólica (PAs) e diastólica (PAd) foram realizadas com as pacientes na posição sentada, com prévia medida do perímetro do braço e correção dos valores quando necessário, de acordo com as tabelas que corrigem a pressão arterial pela circunferência do braço.

Com as pacientes em roupas leves, sem sapatos e em pé, foram realizadas as seguintes medidas: pesagem corporal em balança Filizola com aproximação de $0,1 \mathrm{~kg}$, altura em estadiômetro de madeira colocado na parede com aproximação de $0,1 \mathrm{~cm}$ e circunferências de cintura e quadris em duplicata $\mathrm{com}$ aproximação de $0,1 \mathrm{~cm}$, utilizando-se fita plástica não elástica de $0,7 \mathrm{~cm}$ de largura. De acordo com as normas do Consenso de Airlie VA, cm 1985 (20), a circunferência de cintura foi considerada a menor medida horizontal entre as costelas e as cristas ilíacas e a circunferência dos quadris foi medida em nível de maior largura dos mesmos, em geral na região dos grandes trocânteres. Todas as medidas foram feitas pelo mesmo examinador. A RCQ foi calculada como a razão entre os valores da cintura e os valores dos quadris. O IMC foi calculado como o peso $(\mathrm{kg})$ dividido pela altura $(\mathrm{m})$ ao quadrado $\left(\mathrm{IMC}=\mathrm{kg} / \mathrm{m}^{2}\right.$ ).

O percentual de gordura corporal (\%GC) foi determinado pelo método de Impedância Bioelétrica. Com aparelho portátil de marca RJL, modelo $101 \mathrm{Q}$, a resistência e a reactância à passagem de corrente elétrica de $50 \mathrm{kHz}$ foram medidas, estando a paciente em decúbito dorsal, após esvaziamento da bexiga, com membros superiores e inferiores afastados do tronco e os eletrodos colocados na mão e no pé do lado direito. A equação de Gray et al. (21) foi utilizada para o cálculo de massa magra e percentual de gordura corporal. Consideramos obesas as pacientes com per- 
centual de gordura corporal acima de $31 \%$, de acordo com Lohman (22).

As amostras de sangue foram colhidas após 12 horas de jejum para as dosagens de glicose, insulina, colesterol total e frações e triglicerídeos; as dosagens foram realizadas pelo Laboratório Central do $\mathrm{HC}$ FMUSP.
Os exames por TC foram feitos no período matutino, com equipamento Philips TOMOSCAN LX, na Divisão de Imagem do Instituto do Coração, após calibragem diária. Os parâmetros radiográficos para investigação abdominal foram $120 \mathrm{kV}, 200 \mathrm{~mA}$, tempo de exposição de 1,9 segundos, espessura de corte de $10 \mathrm{~mm}$. Os exames foram feitos na paciente

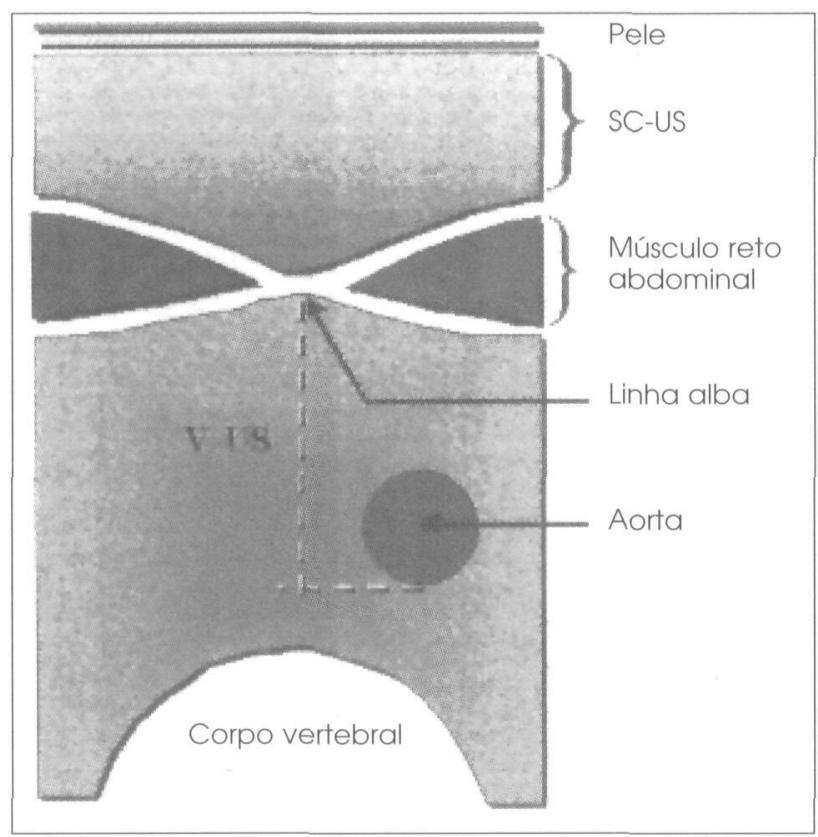

(A)

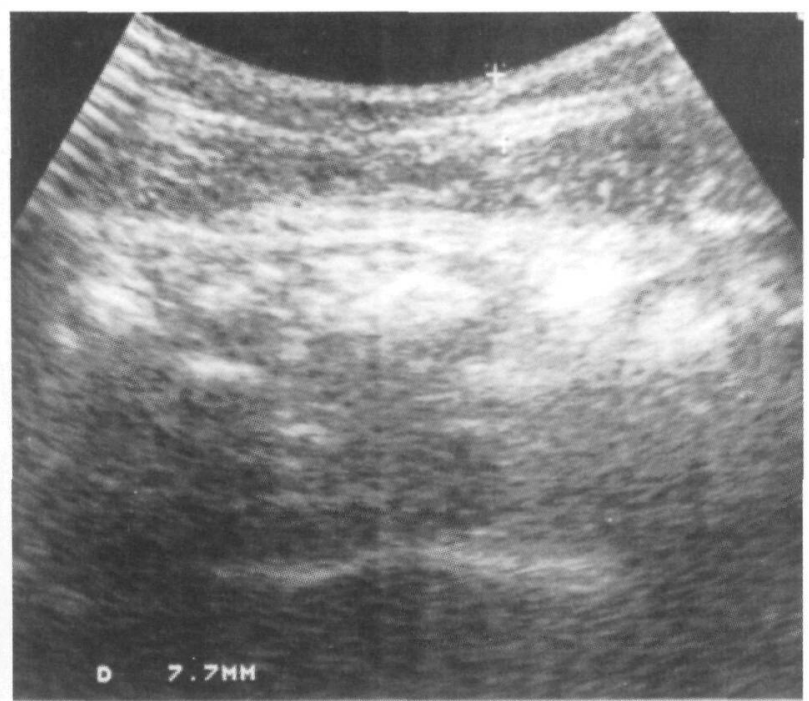

(B)

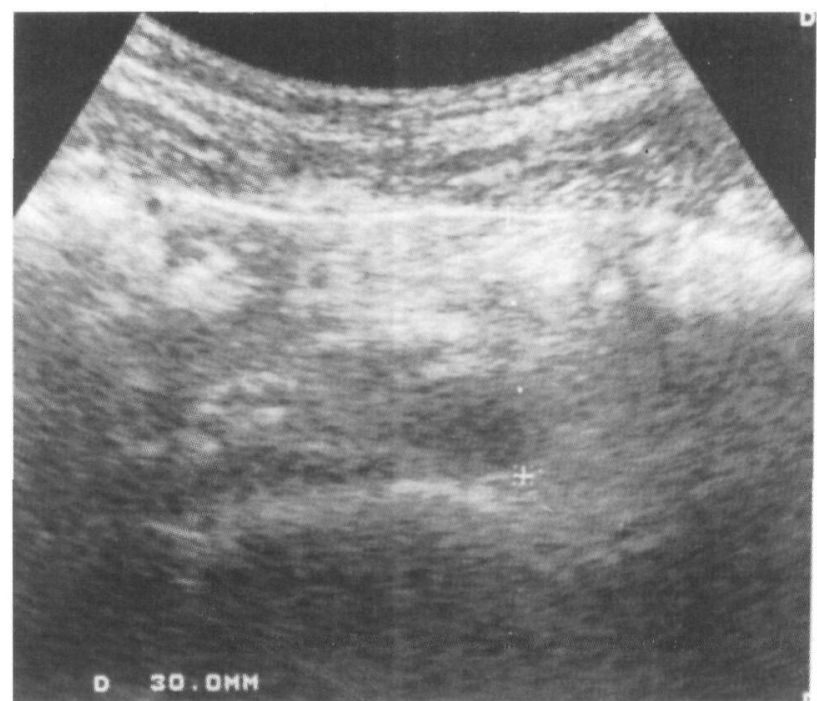

(C)

Figura 1. Diagrama das medidas de espessura intra-abdominal e subcutânea (A) Ultra-Sonografia com os locais de medição assinalados: espessura subcutânea (B) e espessura intra-abdominal (C). 
em posição supina com os braços estendidos acima da cabeça. A imagem abdominal foi obtida $\mathrm{em}$ nível de L4-L5, após escanograma lateral para a identificação precisa da localização. A área total do tecido adiposo abdominal (T-TC) foi calculada após a marcação da área desejada com um cursor eletrônico e o número de "pixels" foi determinado dentro do intervalo de densidade do tecido adiposo ( -250 a $50 \mathrm{HU}$ ). A área de gordura visceral (V-TC) foi determinada pelo delineamento da cavidade abdominal dentro da área circundada pela musculatura abdominal, incluindo o tecido adiposo retroperitonial, mesentérico e omental. O tecido adiposo subcutâneo (SC-TC) foi obtido subtraindo-se a área intra-abdominal da área de tecido adiposo abdominal total. $\mathrm{O}$ índice $\mathrm{V} / \mathrm{S}$ foi calculado como a razão entre as áreas de gordura visceral e de gordura subcutânea. O diâmetro sagital foi obtido através da imagem congelada gerada pelo computador, tomando-se como pontos de referência a parede anterior e a posterior do abdome, no mesmo corte onde foram realizadas as determinações das áreas abdominais.

As medidas de espessura de tecido adiposo foram obtidas em equipamento de US de marca TOSHIBA, modelo SSA 250 A (que utiliza modo B de representação de imagem), na Divisão de Imagem do Instituto do Coração, no mesmo dia da avaliação pela TC. As medidas foram realizadas em triplicata, sempre pelo mesmo operador, com transdutor convexo eletrônico de $3,5 \mathrm{MHz}$ na paciente em jejum e em decúbito dorsal, em região imediatamente superior à cicatriz umbilical, na linha xifoumbilical. A leitura foi feita diretamente de imagens congeladas na tela. Considerou-se espessura subcutânea (SC-US) a medida entre pele e face externa do músculo reto abdominal e espessura intraabdominal (V-US) a medida entre face interna do músculo reto abdominal e parede posterior da aorta (Figura 1).

A reprodutibilidade do método foi testada por um mesmo operador $\mathrm{cm} 10$ indivíduos, através de medições em triplicata, em dois dias diferentes.

Para a avaliação de reprodutibilidade foi feito o teste $t$ de Student e calculado o coeficiente de variação das diferenças. Para o estudo da interdependência linear das variáveis foi utilizado o coeficiente de correlação de Pearson e aplicado o teste de significância estatística. Para a equação preditiva foi utilizado o teste de Regressão Múltipla com inclusão das variáveis passo-a-passo. Os parâmetros em tercis foram obtidos por análise de variância com fator aleatório. Uma vez detectada significância estatística na análise de variância, foi realizado o teste de comparação múltipla de Tukey para detectar as diferenças entre os níveis de fatores. O nível de significância adotado como base para decisão foi de menor ou igual a $5 \%$.

\section{RESULTADOS}

O teste de reprodutibilidade intra-examinador das medidas obtidas através da US apresentou coeficientes de variação de $5 \%$ e $2,6 \%$ para tecido adiposo subcutâneo e intra-abdominal, respectivamente.

As características do grupo estudado estão nas Tabelas 1 (médias e desvios padrão). Na Tabela 2 estão as correlações entre os parâmetros estudados. A espessura intra-abdominal medida pela US mostrou correlações significantemente positivas com IMC, diâmetro sagital, \%GC, área visceral e $\mathrm{V} / \mathrm{S}$. A espessura subcutânea mostrou correlações positivas significantes com IMC, cintura, RCQ, diâmetro sagital, \%GC e com áreas subcutânea e total. A área visceral apresentou correlações positivas e significantes com IMC, cintura, diâmetro sagital, \%GC, espessura intra-abdominal, área adiposa total e $\mathrm{V} / \mathrm{S}$. Entrc os parâmetros bioquímicos, o único a ter correlações significantes foi a variável

Tabela 1. Características do grupo de mulheres estudadas (médias e desvios padrão) $n=29$.

\begin{tabular}{|c|c|c|c|c|}
\hline PARÂMETRO & MÉDIA & $\begin{array}{c}\text { DESVIO } \\
\text { PADRÃO }\end{array}$ & MÍNIMO & MÁXIMO \\
\hline IDADE (anos) & 33,97 & 9,21 & 16 & 50 \\
\hline PAs $(\mathrm{mmHg})$ & 127,07 & $\pm 15,09$ & 100 & 160 \\
\hline $\mathrm{PAd}(\mathrm{mmHg})$ & 82,07 & $\pm \quad 10,57$ & 60 & 100 \\
\hline PESO (kg) & 78,82 & $\pm \quad 10,77$ & 55,5 & 101,00 \\
\hline ALTURA $(\mathrm{cm})$ & 158.79 & $\pm \quad 6,86$ & 147,5 & 173,3 \\
\hline $\operatorname{IMC}\left(\mathrm{kg} / \mathrm{m}^{2}\right)$ & 31,2 & $\pm \quad 3,24$ & 24,02 & 37,45 \\
\hline CINTURA $(\mathrm{cm})$ & 92,14 & \pm 11.49 & 65,00 & 115,00 \\
\hline QUADRIL $(\mathrm{cm})$ & 107,76 & $\pm 8,73$ & 87,00 & 122,00 \\
\hline$R C Q$ & 0,86 & $\pm 0,09$ & 0.66 & 1,13 \\
\hline$\% G C$ & 41,72 & 3,84 & 34 & 50 \\
\hline $\mathrm{DS}(\mathrm{cm})$ & 22,11 & 2,7 & 16,0 & 29,0 \\
\hline V-TC $\left(\mathrm{cm}^{2}\right)$ & 75,06 & $\pm \quad 32,48$ & 30,74 & 189,91 \\
\hline $\mathrm{SC}-\mathrm{TC}\left(\mathrm{cm}^{2}\right)$ & 365,66 & $\pm 103,03$ & 183.68 & 597,37 \\
\hline $\mathrm{T}-\mathrm{TC}\left(\mathrm{cm}^{2}\right)$ & 440,71 & $\pm 109,87$ & 233,64 & 652,55 \\
\hline$V / S$ & 0,22 & $\pm 0,11$ & 0,08 & 0,59 \\
\hline V-US (mm) & 63,31 & $\pm 14,14$ & 40.5 & 93,0 \\
\hline SC-US $(\mathrm{mm})$ & 38,53 & $\pm 13,16$ & 20,0 & 70,0 \\
\hline $\mathrm{COL}(\mathrm{mg} / \mathrm{dL})$ & 191,90 & $\pm \quad 37,39$ & 137,00 & 263,00 \\
\hline $\mathrm{HDL}(\mathrm{mg} / \mathrm{dL})$ & 54.07 & $\pm 14,13$ & 32,00 & 95,00 \\
\hline TRIG $(\mathrm{mg} / \mathrm{dL})$ & 105,52 & $\pm 40,64$ & 36,00 & 197,00 \\
\hline INS $(\mathrm{mUJ} / \mathrm{mL})$ & 18,89 & \pm 8.67 & 5,00 & 38,4 \\
\hline GLIC $(\mathrm{mg} / \mathrm{dL})$ & 92.45 & $\pm 10,80$ & 67,00 & 122,00 \\
\hline
\end{tabular}


Tabela 2. Correlações (r) entre os parâmetros antropométricos, áreas e espessuras adiposas $(n=29)$.

\begin{tabular}{lcccccccccc}
\hline IMC & $\begin{array}{c}\text { CIN } \\
\text { TURA }\end{array}$ & RCQ & DS & $\begin{array}{c}\text { V-US } \\
\text { SC- } \\
\text { US }\end{array}$ & $\begin{array}{c}\text { V- } \\
\text { TC }\end{array}$ & $\begin{array}{c}\text { SC- } \\
\text { TC }\end{array}$ & T-TC & V/S \\
IMC & 1 & $0,79^{*}$ & $0,41^{*}$ & $0,72^{*}$ & $0,42^{*}$ & $0,74^{*}$ & $0,50^{*}$ & $0,74^{*}$ & $0,84^{*}$ & 0,02 \\
Cintura & $0,79^{*}$ & 1 & $0,73^{*}$ & $0,69^{*}$ & 0,17 & $0,76^{*}$ & $0,52^{*}$ & $0,68^{*}$ & $0,79^{*}$ & 0,11 \\
RCQ & $0,41^{*}$ & $0,73^{*}$ & 1 & $0,47^{*}$ & 0,01 & $0,49^{*}$ & 0,35 & $0,40^{*}$ & $0,48^{*}$ & 0,11 \\
DS & $0,72^{*}$ & $0,69^{*}$ & $0,47^{*}$ & 1 & $0,48^{*}$ & $0,57^{*}$ & $0,57^{*}$ & $0,66^{*}$ & $0,79^{*}$ & 0,13 \\
\%GC & $0,76^{*}$ & $0,55^{*}$ & $0,43^{*}$ & $0,81^{*}$ & $0,52^{*}$ & $0,48^{*}$ & $0,59^{*}$ & $0,49^{*}$ & $0,64^{*}$ & 0,20 \\
V-US & $0,42^{*}$ & 0,17 & 0,01 & $0,48^{*}$ & 1 & 0,12 & $0,64^{*}$ & 0,07 & 0,25 & $0,52^{*}$ \\
SC-US & $0,74^{*}$ & $0,76^{*}$ & $0,49^{*}$ & $0,57^{*}$ & 0,12 & 1 & 0,14 & $0,79^{*}$ & $0,78^{*}$ & $(0,29)$ \\
V-TC & $0,50^{*}$ & $0,52^{*}$ & 0,35 & $0,57^{*}$ & $0,64^{*}$ & 0,14 & 1 & 0,06 & 0,35 & $0,82^{*}$ \\
SC-IC & $0,74^{*}$ & $0,68^{*}$ & $0,40^{*}$ & $0,66^{*}$ & 0,07 & $0,79^{*}$ & 0,06 & 1 & $0,96^{*}(0,47)^{*}$ \\
T-TC & $0,84^{*}$ & $0,79^{*}$ & $0,48^{*}$ & $0,79^{*}$ & 0,25 & $0,76^{*}$ & 0,35 & $0,96^{*}$ & 1 & $(0,20)$ \\
V/S & 0,02 & 0,11 & 0,11 & 0,13 & $0,53^{*}$ & $(0,29)$ & $0,82^{*}$ & $(0,47)^{*}(0,20)$ & 1 \\
\hline
\end{tabular}

$* 0,05$

IMC: Indice de Massa Corporal

RCQ: Relação Cintura-Quadrll

DS: Diâmetro Sagital

\%GC: Percentagem de Gordura Corporal

V-US: Espessura Intra-abdominal medida pela US

SC-US: Espessura Subcutânea medida pela US

V-TC: Área Visceral medida pela TC

SC-TC: Área Subcutânea medida pela TC

T-TC: Área Adiposa Abdominal Total medida pela TC

V/S: Relação entre Área Visceral e Área Subcutânea

triglicerídeos (Tabela 3). As correlações foram positivas com cintura, diâmetro sagital, espessura intra-abdominal e área adiposa visceral. Os níveis tensionais mostraram correlações lineares positivas significantes com a espessura intra-abdominal (Tabela 3).

Para a predição da área de gordura intraabdominal foi realizada a análise de regressão múltipla passo-a-passo, utilizando-se como variável dependente a área adiposa visceral medida pela TC e como variáveis independentes as espessuras subcutânea e intra-abdominal medidas por US, IMC, cintura, quadril, RCQ, \%GC, idade, peso, altura e diâmetro sagital.

A tríade de variáveis de maior correlação com a área de gordura visceral foi a composta pelas medidas de espessuras intra-abdominal e subcutânea obtidas pela US e a medida de cintura (coeficiente de correlação múltipla de 0,86 ).

A melhor equação de regressão encontrada foi:

$\mathrm{V}-\mathrm{TC}=18.000+250,2$ cintura $+129,2(\mathrm{~V}-\mathrm{US})-$ 148,6 (SC - US)

$\mathrm{R}^{2}=0,74$

Os parâmetros IMC, cintura, RCQ, DS, VUS e $\mathrm{V} / \mathrm{S}$ foram divididos em tercis (Tabela 4), tendo como referência os valores de área adiposa intra-abdominal de $56 \mathrm{~cm}^{2}$ e $81 \mathrm{~cm}^{2}$. A tabela
Tabela 3. Correlações (r) dos parâmetros antropométricos, áreas adiposas, espessuras adiposas, exames bioquimicos e niveis tensionais $(n=29)$.

$$
\text { IMC } \underset{\text { TURA }}{\text { CIN }} \text { RCQ DS V-US } \begin{gathered}
\text { SC- } \\
\text { US }
\end{gathered}
$$

$\begin{array}{lllllllllll}\text { COL } & 0,05 & (0,08) & (0,19) & 0,30 & 0,19 & (0,10) & 0,13 & 0,08 & 0,11 & 0,02\end{array}$

HDL $(0.20)(0.37)(0.37) \quad 0 \quad(0.06)(0.22)(0.29)(0.08)(0.16)(0.22)$

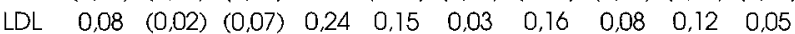

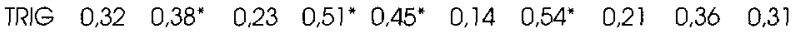

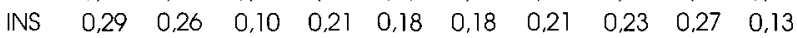



$\begin{array}{lllllllllll}\text { PAs } & 0,3 & 0,01 & (0,10) & 0,16 & 0,45^{*} & 0,05 & 0,28 & 0 & 0,08 & 0,17\end{array}$

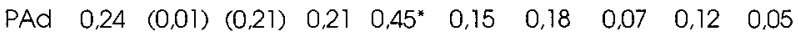

$* 0,0,05$

IMC: İndice de Massa Corporal

$\mathrm{RCQ}$ : Relação Cintura-Quadril

DS: Diâmetro Sagital

\%GC: Percentagem de Gordura Corporal

V-US: Espessura Intra-abdominal medida pela US

SC-US: Espessura Subcutânea medida pela US

V-TC: Área Visceral medida pela TC

SC-TC: Área Subcutânea medida pela TC

T-TC: Área Adiposa Abdominal Total medida pela TC

V/S: Relação entre Área Visceral e Área Subcutânea

PAs: Pressão Arterial Sistólica

PAd: Pressão Arterial Diastólica

Tabela 4. Médias e erros padrão dos valores de vários parâmetros divididos por tercis de V-TC $(n=29)$.

\begin{tabular}{lccc}
\hline V-TC & $\begin{array}{c}<6 \mathrm{~cm}^{2} \\
n=9\end{array}$ & $\begin{array}{c}56-81 \mathrm{~cm}^{2} \\
n=10\end{array}$ & $\begin{array}{c}>81 \mathrm{~cm}^{2} \\
n=10\end{array}$ \\
IMC $\left(\mathrm{kg} / \mathrm{m}^{2}\right)$ & $28,96 \pm 1,17 \mathrm{a}$ & $31,84 \pm 0,49 \mathrm{~b}$ & $32,58 \pm 1,08 \mathrm{~b}$ \\
CINTURA (cm) & $83,30 \pm 3,75 \mathrm{a}$ & $96,20 \pm 2,94 \mathrm{~b}$ & $96,00 \pm 3,16 \mathrm{~b}$ \\
RCQ & $0,78 \pm 0,03 \mathrm{a}$ & $0,90 \pm 0,03 \mathrm{~b}$ & $0,87 \pm 0,02 \mathrm{~b}$ \\
DS (cm) & $20,03 \pm 0,91 \mathrm{a}$ & $22,77 \pm 0,28 \mathrm{~b}$ & $23,31 \pm 0,94 \mathrm{~b}$ \\
V-US (mm) & $53,39 \pm 2,87 \mathrm{a}$ & $58,20 \pm 3,88 \mathrm{a}$ & $77,35 \pm 2,45 \mathrm{~b}$ \\
V/S & $0,16 \pm 0,02 \mathrm{a}$ & $0,17 \pm 0,01 \mathrm{a}$ & $0,32 \pm 0,04 \mathrm{~b}$ \\
\hline
\end{tabular}

As médias marcadas com letras diferentes são significantemente diferentes.

$p<0,05$

mostra que o IMC, a cintura, a RCQ, e o DS só discriminaram o $1^{\circ}$ tercil. Por sua vez, os valores de US e o V/S discriminaram apenas o $3^{\circ}$ tercil. A correlação entre os valores de espessura intraabdominal pela US e área intra-abdominal pela TC foi negativa no primeiro tercil $(r=-0,05)$, tornando-se positiva e gradativamente maior nas outras duas classes, $\left(r=0,12\right.$ e $r=0,23$, no $2^{\circ}$ e $3^{\circ}$ tercis, respectivamente).

Observou-se que 4 pacientes distribuídas no $2^{9}$ tercil pela US foram classificadas no $1^{\circ}$ tercil pela $\mathrm{TC}$, 7 pacientes distribuídas no $1^{\circ}$ e $3^{\circ}$ tercis pela US estavam no $2^{\circ}$ tercil pela TC e somente 3 pacientes do 
$3^{\circ}$ tercil da TC foram classificadas no $2^{Q}$ tercil pela US, de acordo con1 o resumo abaixo:

\begin{tabular}{lccc} 
& \multicolumn{3}{c}{ V-US } \\
V-TC & I tercil & II tercil & III tercil \\
I tercil & 5 & 4 & 0 \\
II tercil & 5 & 3 & 2 \\
III tercil & 0 & 3 & 7
\end{tabular}

\section{DISCUSSÃO}

O objetivo principal do nosso estudo foi testar a viabilidade da US em substituir a TC na determinação da gordura visceral, comparando os resultados obtidos pelos dois métodos.

Em 1990, Armellini et al. (23) sugeriram o uso da US como um método direto na determinação da adiposidade intra-abdominal. Os autores mediram a distância entre parede interna do músculo reto abdominal e a parede posterior da aorta, em 50 mulheres obesas, c compararam os resultados com áreas viscerais obtidas por TC, obtendo boa correlação entre os métodos.

Nas publicações analisadas, a reprodutibilidade intra-observador da US para as medidas de espessura intra-abdominal variou entre $0,41 \%$ e $13 \%$ (24-27). As discrepâncias entre os resultados foram atribuídas às diferenças entre as técnicas utilizadas, população escolhida e nível de treinamento dos opcradores. Obtém-se melhor reprodutibilidade quando o exame é fcito por operador bem treinado, com bom controle de pressão do transdutor sobre o abdome do paciente e em indivíduos mais obesos (27). No presente trabalho, o CV encontrado para $\mathrm{V}$-US foi de $2,6 \%$, o que demonstra uma boa precisão do método.

Encontramos correlação de $64 \%$ entre as medidas de tecido adiposo intra-abdominal obtidas pelos dois métodos, US e TC. Entre os mesmos parâmetros, Armellini et al. (23) relataram correlações de $67 \%$ quando descreveram o método, em 1990 , e de $74 \%$ quando propuseram equações preditivas, em 1993 (28). No estudo dessas duas publicações, pudemos observar que, cntre $1990 \mathrm{e}$ 1993, houve modificação na técnica de medição da gordura visceral. O medidor eletrônico foi deslocado $5 \mathrm{~cm}$ acima da cicatriz umbilical, e um dos pontos de referência para a medida intra-abdominal foi transferido de parede posterior da aorta para sua parede anterior. Tais modificações, aliadas a um maior treinamento, podem ter oferecido maior acurácia ao método. Em nosso estudo, optamos pela técnica descrita por Armellini et al. em 1990, como também o fez Bellisari et al. (25) em 1993. Deu-se a escolha da primeira técnica por acreditarmos que os valores de espessura intra-abdominal por ela determinados são mais representativos da quantidade de gordura visceral.

Nossos achados evidenciaram que, entre todos os parâmetros analisados, a US foi o método mais acurado para determinar a quantidade de tecido adiposo visceral.

Estes resultados foram confirmados através da análise de regressão múltipla, onde V-US foi a primeira variável independente a aparecer na equação de predibilidade para tecido adiposo visceral. Esse parâmetro forma com SC-US e cintura a tríade de variáveis com maior coeficiente de correlação múltipla com a V-TC $(\mathrm{R}=0,86)$.

A equação, incluindo as três variáveis, pode explicar $74 \%$ da variação da quantidade de gordura abdominal obtida pela TC $\left(\mathrm{R}^{2}=0,74\right)$.

$\mathrm{Na}$ verificação do comportamento da US intra-abdominal c das medidas antropométricas na caracterização dos grupos com quantidades diferentes de tecido adiposo visceral, a amostra foi dividida em tercis de áreas de gordura visceral obtidas pela TC. Através da análise de variância, foram comparados os três grupos. Nenhum dos parâmetros conseguiu identificar com clareza os três tercis de gordura visceral. No entanto, notamos que, à medida que cresceu a área de gordura intraabdominal, foi maior a correlação entre a US e a TC. Concordamos com Tornaghi et al. (27), quando comentam que fontes de crro relevantes na obtençào de V-US - como, por exemplo, dificuldades em se discriminar os pontos de referência ecográficos nos indivíduos mais magros - se tornam menos importantes à medida em que a quantidade de tecido adiposo aumenta.

A população estudada não apresentou, no geral, níveis bioquímicos compatíveis com risco para doenças cardiovasculares. No entanto, pudemos observar correlações significantes entre $\mathrm{V}$-US, níveis tensionais e triglicerídeos.

Os resultados deste estudo demostram que a US é um método preciso, acurado e que pode ser utilizado para a determinação da gordura visceral. É seguro, prático e não possui os aspectos negativos da TC, podendo ser repetido quando se fizer necessário.

Partimos do princípio de que a obesidade visceral é um fator de risco tanto de morbidade quanto de mortalidade cardiovascular, independente da dis- 
lipidemia, da hipertensão arterial e do diabetes melito a ela associados. Acreditamos que estudos posteriores, com amostragem maior de homens e mulheres em diferentes faixas etárias, poderão estabelecer valores de espessura intra-abdominal preditivos de risco, da mesma forma como acontece com os valores de glicemia, colesterol e frações, triglicerídeos e níveis pressóricos.

\section{REFERÊNCIAS}

1. Van Itallie TB. Health implications of overweight and obesity in the United States. Ann Intern Med 1985; 103:983-8.

2. Manson JE, Willett WC, Stampfer MJ, Colditz GA, Hunter DJ. Hankinson SE, et al. Body weight and mortality among women. N Engl J Med 1995;333:677-85

3. Stamler RA, Stamler J, Riedlinger WF, Algera $G$, Roberts $\mathrm{RH}$. Weight and blood pressure findings in hypertension screening of 1 million Americans, JAMA 1978:240:1607-10.

4. Felber JP. From obesity to diabetes. Pathophysiological considerations. Int $J$ Obes Relat Metab Disord 1992; $16: 937-52$.

5. Stampfer MJ, Maclure KM, Colditz GA, Manson JE, Willet WC. Risk of symptomatic gallstones in women with severe obesity. Am J Clin Nutr 1992;55:652-8.

6. Garfinkel L. Overweight and mortality. Cancer 1986;58:1826-9.

7. Harris T, Cook EF, Garrison R, Higgins M, Kannel W, Goldman L. Body mass index and mortality among nonsmoking older persons: The Framingham Heart Study. JAMA 1988;259:1520-4.

8. Vague J. The degree of masculine differentiation of obesities: a factor determining predisposition to diabetes, atherosclerosis, gout and uric calculus disease. Am J Clin Nutr 1956:4:20-34.

9. Kissebah AH, Vydelingum N, Murray R, Evans DJ, Hartz AJ, Kalkhoff RK, et al. Relation of body fat distribution to metabolic complications of obesity. J Clin Endocrinol Metab 1982;54:254-60.

10. Larsson B, Svardsudd K, Welin L, Wilhelmsen L, Björntorp $P$. Tibblin $G$. Abdominal adlpose tissue distribution, obesity, and risk of cardiovascular disease and death: 13 year follow up of participants in the study of men born in 1913. BMJ 1984;288:1401-4.

11. Ashwell M, Chinn S, Stalley S, Garrow JS. Female fat distribution - a simple classification based on two circumference measurements. Int J Obes 1982;6: 143-52.

12. Tokunaga $K$, Matsuzawa $Y$, Ishikawa $K$, Tarui $S$. A novel technique for the determination of body fat by computed tomography. Int J Obes 1983:7:437-45.

13. Fujioka S, Matsuzawa Y, Tokunaga K, Tarui S. Contribu- tion of intra-abdominal fat accumulation to the impairment of glucose and lipid Metabolism in human obesity. Metabolism 1987:36:54-7

14. Després JP, Nadeau A, Tremblay A, Ferland M, Lupien PJ. Role of deep abdominal fat in the association between regional adipose tissue distribution and glucose tolerance in obese women. Diabetes $1990 ; 38: 304-9$.

15. Després JP, Moorjani S, Lupien PJ, Tremblay A, Nadeau A, Bouchard C. Regional distribution of body fat, plasma lipoproteins, and cardiovascular disease. Arteriosclerosis $1990 ; 10: 497-511$

16. Caprio S, Hyman LD, Mccarthy S, Lange R, Bronson M, Tamborlane WV. Fat distribution and cardiovascular risk factors in obese adolescent girls: importance of the intra-abdominal fat depot. Am J Clin Nutr 1996:64:12-7

17. Lerário AC, Bosco A, Rocha M, Santomauro AT, Luthold W. Gianella D, et al. Risk factors in obese women, with particular reference to visceral fat component. Diabetes Metab 1997:23:68-74.

18. Rocha MS. Contribuição da tomografia computadorizada ao estudo da distribuição de gordura abdominal em mulheres obesas. São Paulo, 1993. Tese (Doutorado) - Faculdade de Medicina, Universidade de São Paulo.

19. Van Der Koy K, Seidell JC. Techniques for the measurement of visceral fat: a practical guide. Int J Obes Relat Metab Disord 1993; $17: 187-3$.

20. Lohman TG, Roche AF, Martorell R. Anthropometric Standardization Reference Manual. Champaign, IL, Human Kinetics, 1988.

21. Gray DS, Bray GA, Gemayel N, Kaplan K. Effect of obesity on bioelectrical impedance. Am J. Clin Nutr 1989:50:255-60.

22. Lohman TG. Advances in Body Composition Assessment. Champaign, IL, Human Kinetics, 1992

23. Armellini F, Zamboni M, Rigo L, Todesco T, BergamoAndreis IA, Procacci $C$, et al. The contribution of sonography to the measurement of intra-abdominal fat. J Clin Ultrasound 1990;18:563-7.

24. Armellini $F$, Zamboni $M$, Rigo $L$, Bergamo-Andreis $I A$, Robbi $R$, De Marchi $M$, et al. Sonography detection of small intra-abdominal fat variations. Int $\mathrm{J}$ Obes 1991; 15:847-52.

25. Bellisari A, Roche AF, Siervogel RM. Reliability of B-mode ultrasonic measurements of subcutaneous adipose tissue and intra-abdominal depth: comparisons with skinfold thicknesses. Int J Obes Relat Metab Disord 1993; 17:475-80.

26. Armellini F, Zamboni M, Casteli S. Rocco M. Mino A, Turcato $E$, et al. Measured and predicted total and visceral adipose tissue in women. Correlations with metabolic parameters. Int J Obes Relat Metab Disord $1994 ; 18: 641-7$ 
27. Tornaghi $G$, Raiteri R, Pozzato C, Rispoili A, Bramani $M$. Cipolat $M$, et al. Anthropometric or ultrasonic measurements in assessment of visceral fat? A comparative study. Int J Obes Relat Metab Disord 1994; 18:771-5.

28. Armellini $F$, Zamboni $M$, Robbi $R$, Todesco $T$, Rigo $L$, Bergamo-Andreis IA, et al. Total and intra-abdominal fat measurements by ultrasound and computerized tomography. Int J Obes Relat Metab Disord 1993; 17:209-14.

\section{Endereço para correspondência:}

Rosana Bento Radominski

Av. Visconde de Guarapuava 5085, apto 1101

80240-010 Curitiba. PR

E-mail: rbr@uol.com.br 